\title{
Principle design of regional energy optimal allocation
}

\author{
Congcong $\mathrm{Li}^{1 *}$, Qing Wang ${ }^{1}$, Hongxia Zhu ${ }^{1}$, Xinping Wang ${ }^{1}$, Chao $\mathrm{Yu}^{1}$, Zhixue Wang ${ }^{2}$, Wei Guo ${ }^{2}$ \\ ${ }^{1}$ Department of Electrical Engineering and Automation, Qilu University of Technology (Shandong Academy of Sciences), Jinan 250353, \\ China \\ ${ }^{2}$ School of Control Science and Engineering, Shandong University, Jinan 250061, China
}

\begin{abstract}
As the proposal of energy internet and intelligent energy, the utilization of energy and the energy categories has become more and more diverse. This paper redefines the concept of multi energy interconnection network, and divides local energy network, regional energy network and wide area energy network. In the planning and construction of energy interconnection network, the very important work is the optimal allocation of energy. In order to better allocate energy, we need to abide by specific principles, but there are few literatures on energy allocation principles. Taking the regional energy network as the research object, several principles of energy optimal allocation in the regional energy network are designed, and each principle is explained in detail. Finally, principles can provide reference for the planning and construction of regional men.
\end{abstract}

\section{Introduction}

With the development of social economy, people's demand for energy is higher and higher. However, energy reserves will not increase with the increase of people's demand, so how to maximize the use of effective energy and maintain the sustainability of the whole energy system has become the focus of attention in recent years. "Smart energy" and "energy Internet" have become research hotspots. Reference [1] gives a preliminary definition of energy Internet, outlines the basic architecture and composition of energy Internet. It discusses the core issues and possible problems in related fields. Reference [2] makes a good analysis of energy Internet and smart energy. It divides energy Internet into global energy Internet, broad energy Internet and narrow energy Internet, and analyzes the concept of each kind of energy Internet in detail. Reference [3-5] introduces the framework design and topology model design of energy Internet in detail. Reference [6-7] studies the comprehensive evaluation of energy Internet and smart grid.

With the development of the research on energy Internet and smart energy, as an important part of "energy", people put forward higher requirements for it in the form of energy, energy quality, sustainability or economy. Therefore, how to optimize the energy allocation of a region has become a necessary work for the construction of the regional energy network. At present, the optimal allocation of energy is mainly for a certain aspect of research. There is no systematic principle of energy allocation. Reference [8] studies the security of China's regional energy supply, and puts forward the path and strategy for the security optimization of regional energy supply. Reference [9] studies the optimal allocation of energy in small towns, and preliminarily discusses the policies and measures for the optimal allocation of energy in small towns.

This paper considers the requirements of energy security, reliability, economy and sustainability of energy network, which aims to study the optimal allocation of regional energy. It designs the principle of energy optimal allocation suitable for general situation. This paper puts forward the principle of regional energy optimal allocation and analyzes the relationship between different principles. It gives the flow chart of energy optimal allocation.

\section{Basic concepts of multi-energy interconnection network}

\subsection{Energy interconnection network and its hierarchy}

Multi energy interconnection network is a comprehensive energy utilization network integrating production, transmission, conversion and utilization of various energy forms. Different forms of energy flow in their own unique energy network. And different energy networks are interconnected in a specific form, forming a network of combined utilization of a variety of energy.

Energy network includes local energy network, regional energy network and wide area energy network. However, there seems to be no unified standard to distinguish the concepts of local area, regional area and wide area. Even the same terms mentioned in different literatures represent different concepts, which has caused a lot of trouble to the follow-up research of related directions. Therefore, it is necessary to sort out the

\footnotetext{
* Corresponding author: 1258449230@qq.com
} 
relevant concepts and propose a unified classification standard.

In fact, there are great differences in the planning and construction of energy interconnection networks at different levels, including user complexity, geographical scope, influencing factors, energy consumption, etc.

In this paper, all kinds of energy are discussed on an equal position. And the relationships among different forms of energy and different levels of network are discussed. All kinds of energy forms are to meet the energy needs of users, with only differences in quantity, but no differences in status. Each energy form may have a complete or local energy network. And multiple energy networks can be interconnected through energy conversion. According to the scale of energy consumption and load distribution, the energy network will have different levels. Different levels are related to each other to some extent, and each energy micro-grid in the same level is also related and complementary to each other.

To sum up, men is proposed to build a peer-to-peer interconnection, open sharing, multi-source collaboration, low-carbon and efficient energy utilization network, promote the process of China's energy revolution. It alleviates China's severe energy environment. The key technologies of men include energy management technology, energy conversion technology, advanced energy storage technology, advanced information technology and so on. The core of men is to ensure the overall coordination of multiple energy networks and the comprehensive and efficient utilization of various forms of energy.

\subsection{Concepts of regional energy interconnection network}

This paper mainly studies the regional energy network. The so-called regional energy network basically includes the final types of energy, a large number of users and a large geographical range. It simply considers the human factors and political factors in the target area, and on the basis of fully considering the existing resources in the region. It completes the energy network by comprehensive, integrated and complementary application of various equipment, technologies and systems, and comprehensively considering the energy outside the region and adjusting measures to local conditions. The whole process of production, supply, transmission and distribution, use and emission of regional energy interconnection network.

The area can be as small as two adjacent buildings, or it can be large to a city, island, etc. From the perspective of geographical category, it is a medium scope concept. From the perspective of user category, it breaks through the single user type and belongs to a more complex concept. From the perspective of the category of energy use, it includes the basic end energy form. The regional energy network realizes the interconnection of multiple units and users in one region. The load between different users is complementary to meet different types of energy demand. The regional energy network can basically realize the efficient, economic and sustainable utilization of energy.

Research on regional energy network, including energy supply side and energy demand side. The energy supply side includes both traditional energy and renewable energy, including a single form of energy supply and a variety of energy supply forms of CCHP, as well as the effective allocation between them. On the energy demand side, the regional energy network can meet the user's demand for cold, heat, electricity, steam and other terminal energy, thus providing favorable conditions for the centralized management of all kinds of energy.

\section{Principles of energy optimal allocation in regional men}

For the planning and construction of men, energy allocation is one of the important parts. A reasonable energy allocation is the basis of clean, safe and sustainable operation of men. Due to the differences between different regions and different energy forms in the same region, there are great differences in the energy optimal allocation of men. Men include various energy forms, such as electricity, cold / heat, natural gas, etc. each energy form has different standards. Based on the requirements of men for safety, reliability, economy and sustainability, this paper designs the principle of regional energy optimization allocation based on the requirements of men for safety, reliability and sustainability, considering the differences of different regions, different energy and load forms.

\subsection{Energy supply and demand balance principle}

$$
\begin{gathered}
P_{1}+P_{2}+P_{3}-P_{\mathrm{b}} \approx P_{\mathrm{c}} \\
E_{1}+E_{2}+E_{3}-E_{\mathrm{b}} \approx E_{\mathrm{c}}
\end{gathered}
$$

Energy supply and demand balance is an important part to ensure the security and reliability of energy network. Energy supply and demand balance includes two parts: power balance and energy balance.

When the power of the system in the period is balanced. The actual production power $\mathrm{P}_{1}, \mathrm{P}_{2}, \mathrm{P}_{3}$ of each equipment, excluding the loss power $\mathrm{P}_{\mathrm{b}}$, is equal to the required power PC of the system. Energy supply refers to the total amount of energy that can be provided in a certain period. According to energy sources, it can be divided into self-production $E_{1}$, external input $E_{2}$ and inventory $\mathrm{E}_{3}$. Energy demand refers to the total amount of energy required by users in a certain period, which can be divided into consumption EC and reserve EB according to energy use.

Too high load will seriously affect the stability of the energy network and cause damage to the equipment. Too low load will cause waste of energy and idle of some units. At present, a lot of energy supply and demand imbalance, the overall fluctuation is large. In order to meet the energy demand of users, it often will increase the input of energy supply units, but this can not really 
solve the problem. Therefore, to ensure the balance of energy supply and demand is one of the necessary requirements in the process of energy optimization.

\subsection{Principles of energy quality assurance}

Energy quality is one of the essential factors to ensure the safe and reliable operation of men. There are two indicators to consider energy quality: energy continuity and energy stability. When the energy network is shut down due to failure or maintenance, how to ensure the continuity of energy supply is an important aspect that must be considered. For the stability, it shows the stability of amplitude and frequency in the aspect of electric energy, and the uniformity and stability of steam flow in the aspect of heating. Therefore, to ensure the continuity and stability of energy is to ensure the quality of energy needs to be considered.

\subsection{Principle of full utilization of energy}

The full utilization of energy is one of the necessary principles to meet the safety, reliability, economy and sustainability of men. In the planning and construction of men, we should allocate energy reasonably according to the regional energy and load, and give full play to the regional energy production capacity. On the premise of ensuring energy demand, reduce energy consumption and maximize energy utilization. The full use of energy improves the economy to a certain extent, and also meets the requirements of sustainable use of energy.

In addition to maximizing the utilization of single energy form, the full utilization of energy also includes the full utilization of different energy forms. On the basis of traditional energy, reasonable use of renewable energy, such as wind energy, solar energy and biomass energy, constitute a multi energy complementary energy supply system, realize electricity, heat and cold combined supply. It not only can make full use of energy and improve energy efficiency, but also can reduce the shortcomings of low quality of single energy supply. So it can meet the requirements of safety and reliability. In order to alleviate the pressure on the environment caused by excessive consumption of a certain energy.

\subsection{Principle of rational exploitation of energy}

The principle of reasonable exploitation of energy is one of the important parts to meet the sustainable utilization of energy. With the continuous emergence of energy crisis, land desertification, environmental pollution, species extinction and forest area reduction, the state puts forward the strategy of sustainable development. Sustainable development refers to the development that meets the current needs without weakening the ability of future generations to meet their needs. Sustainable development also means the maintenance, rational use and improvement of the natural resource base. Therefore, energy allocation and exploitation should be coordinated with the local ecological environment, and exploitation should be reasonable according to the planning requirements, so as to maintain the sustainability of local energy.

\subsection{Principle of low environmental cost}

The principle of low environmental cost is one of the essential factors to maintain the economy and sustainability of energy utilization. To adhere to the principle of low environmental cost of energy allocation, we need to start from the energy utilization mode and energy types, choose clean energy, improve the utilization rate of energy and reduce the emissions of harmful substances and other measures.

\subsection{Principle of zoning consideration}

The principle of zoning includes two aspects: on the one hand, it is mainly aimed at the rationality of the layout of hardware facilities in the process of economic energy structure design; on the other hand, it is to achieve "local production and local utilization" in the aspect of energy utilization, so as to reduce the energy loss of longdistance energy transmission and ensure the sustainability of energy.

\subsection{Principle of reasonable energy allocation}

Regional men involve a variety of energy forms. During the operation, the energy reserves of different regions can be used as reserves for each other by reasonable collocation of energy forms. The energy reserves of different energy microgrid in the same region can be used as reserves for each other, and different energy forms of the same energy microgrid can be used as reserves for each other. In this way, each single network can have enough energy reserves, which can improve the security and reliability of the energy network, and realize the integration of the whole energy network. The requirement of reserve energy reduces the cost.

\section{Relationship and application of principles}

\subsection{The interrelationship of the principles}

In the optimal allocation of regional energy, due to different levels of consideration, the principles to be observed are also different. Different principles are of different importance to the whole regional men because of different considerations. Some principles are necessary for regional men, and some principles are not necessary for regional men, but they also play an important role, such as improving the economy of energy optimal allocation. According to the importance of energy network, this paper divides the principles of regional energy optimal allocation into two categories: necessary principles and important principles. The specific classification is shown in Figure 1. 


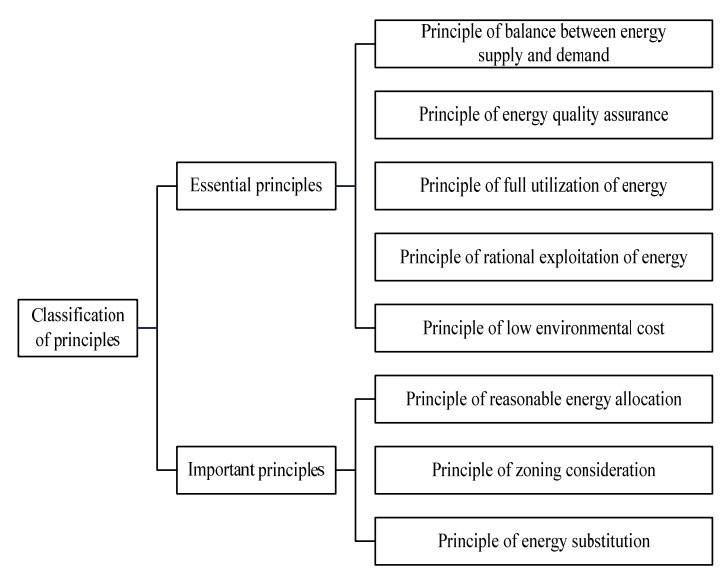

Fig1. Classification principles

The first five principles in Figure 1 are the essential principles in energy allocation. It can be seen that the essential principles are mainly composed of the principles to ensure the safety, reliability and sustainability of regional men. This is enough to see that in the energy allocation of regional men, ensuring the safe and reliable operation of the whole energy network and ensuring the sustainable use of energy are the issues that must be considered. On this basis, economy should be considered.

\subsection{Principle application process}

The principle of energy optimal allocation is applicable to general regional men. This paper gives the flow chart of energy optimal allocation, as shown in Figure 2.

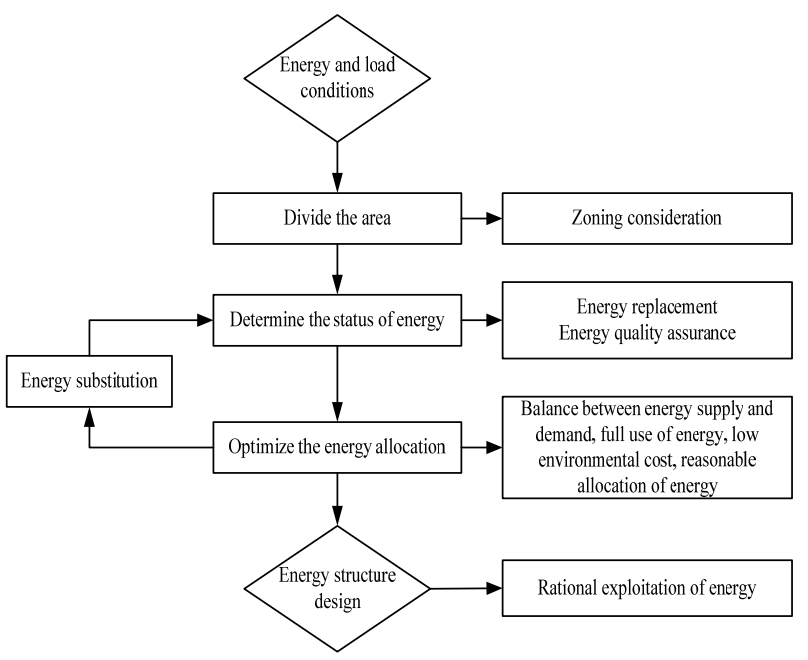

Fig2. Flow chart of optimized allocation of energy

\section{Conclusion}

This paper redefines the concept of multi energy interconnection network, and divides local energy network, regional energy network and wide area energy network. Then, in order to meet the requirements of security, reliability, economy and sustainability of energy network, the principles of energy optimal allocation of regional energy network are proposed. It includs the principle of energy supply and demand balance, the principle of energy quality assurance, the principle of energy full utilization, the principle of reasonable energy exploitation, the principle of low environmental cost, the principle of zoning consideration, the principle of reasonable energy allocation and the principle of energy substitution. Each principle is explained in detail, and different principles are classified. Finally, the principle proposed in this paper can make the maximum use of energy, which can save energy and improve the economic efficiency. It is hoped that these principles can provide reference for the planning and construction of regional men.

\section{References}

1. Yu Y . Editorial for the Special Issue on Smart Grid and Energy Internet. J. Engineering 67 (2020).

2. HUANG Renle, PU Tianjiao, LIU Kewen, et al. Design of hierarchy and functions of regional energy Internet and its demonstration applications. J. Automation of Electric Power Systems 399 (2015).

3. From smart grid to energy Internet: Basic concept and research framework. J. Dianli Xitong Zidonghua/Automation of Electric Power Systems 8 15 (2014).

4. Tian S, Luan W, D Zhang, et al. Technical forms and key technologies on energy Internet. J. Zhongguo Dianji Gongcheng Xuebao/ proceedings of the Chinese Society of Electrical Engineering 35 14 (2015).

5. Sun Q, Wang B , Huang B , et al. The Optimization Control and Implementation for the Special Energy Internet. J. Proceedings of the Csee (2015).

6. Wang W, Wang D, Jia H, et al. Review of Steadystate Analysis of Typical Regional Integrated Energy System Under the Background of Energy Internet. J. Proceedings of the Csee 3612 (2016).

7. Hua, Haochen, Hao, et al. A Class of Control Strategies for Energy Internet Considering System Robustness and Operation Cost Optimization. J. Energies (2018).

8. Xue Jingjing,SHI Jun,SHEN Lei,et al. A research on the regional energy supply security of China. J. China Soft Science 1 (2015).

9. Guangbin,Wang Yong, Yang Xinhai,Huang Yaozhi.College of Resources and Environment Science, Univirersity E C N, et al. Study on Optimal Allocation of Energy in Small Towns. J. China Population, Resources and Environment (2005).

10. Chen Z, Hu H, Wu Y, et al. Stochastic model predictive control for energy management of powersplit plug-in hybrid electric vehicles based on reinforcement learning. J. Energy 21 (2020). 